\title{
Adoption d'une nouvelle méthode de lutte sanitaire en milieu paysan : le pédiluve acaricide/insecticide au Burkina Faso
}

\author{
F. Bouyer ${ }^{1}$ A. Belem ${ }^{2}$ H. Seyni ${ }^{3}$ H. Adakal ${ }^{1}$ \\ R. Lancelot ${ }^{4}$ F. Stachurski ${ }^{4}$ J. Bouyer ${ }^{4,5 *}$
}

\begin{abstract}
Mots-clés
Innovation - Système d'élevage - Lutte anti-insectes - Lutte anti-acariens Intensification - Afrique de l'Ouest.
\end{abstract}

\begin{abstract}
Résumé
Le pédiluve acaricide/insecticide est une nouvelle méthode de lutte intégrée contre les vecteurs en zone subhumide Ouest africaine : son efficacité a été validée par de nombreuses études en situations expérimentales et réelles. Cette invention sanitaire d'origine exogène (proposée par la recherche) a été co-construite avec des groupes d'éleveurs il y a dix ans et a ensuite été diffusée. En tout, 22 pédiluves et 72 éleveurs ont été étudiés. A partir de 97 variables d'adoption concernant la sociologie, les aspects organisationnels, le système d'élevage, l'appréciation de l'outil et les modalités de mise en œuvre, 21 ont été considérées comme actives et sept comme indicateurs d'adoption, suite aux analyses préliminaires. Elles ont été soumises à des analyses multivariées permettant de caractériser trois groupes d'éleveurs, dont l'adoption a été évaluée. Le premier groupe était constitué par les éleveurs modernes de Ouagadougou qui ont bien adopté la méthode. Les éleveurs plus traditionnels de Bobo-Dioulasso ont été séparés en deux groupes dont l'un n'a pas adopté le pédiluve, contrairement au second. Les dix variables discriminant principalement ces groupes ont été analysées. Elles avaient trait au système d'élevage, aux modalités de mise en œuvre de la méthode et à l'appréciation de l'outil. L'appréciation du risque de l'innovation par les éleveurs a été discutée grâce aux critères d'évaluation de l'adoptabilité de Mendras et Forsé. Cette analyse a mis en évidence les variations de perception individuelle des bénéfices et des risques, et le rôle prédominant du réseau socio-technique.
\end{abstract}

1. Centre international de recherche-développement sur l'élevage en zone subhumide (Cirdes), Bobo-Dioulasso, Burkina Faso.

2. Institut du développement rural (IDR), Université polytechnique

de Bobo-Dioulasso (UPB), Bobo-Dioulasso, Burkina Faso.

3. Direction de l'Agriculture et de la sécurité alimentaire (DASA), département Développement rural, ressources naturelles et environnement (DDRE),

Union économique et monétaire Ouest africaine (Uemoa), Ouagadougou, Burkina Faso.

4. UMR Contrôles des maladies animales et émergentes, Centre de coopération internationale en recherche agronomique pour le développement (Cirad), Montpellier, France.

5. Institut sénégalais de recherches agricoles, Laboratoire national d'élevage et de recherches vétérinaires, Hann, Dakar, Sénégal.

* Auteur pour la correspondance

Tél. : +221339510265; fax : +221338323679

E-mail : bouyer@cirad.fr

\section{INTRODUCTION}

Au Burkina Faso, 86 p. 100 de la population exerce une activité d'élevage (principale ou secondaire) : ainsi ce secteur participe à hauteur de 12 p. 100 du produit intérieur brut (PIB) et 19 p. 100 des exportations en valeur (25). En outre, l'agriculture qui assure 40 p. 100 du PIB utilise essentiellement la traction animale pour les principales cultures (coton et céréales). Un des enjeux prioritaires pour le développement de l'élevage bovin est la maîtrise des conditions sanitaires, en particulier la lutte contre la tique Amblyomma variegatum et la lutte contre les trypanosomoses animales, transmises par les glossines, qui entraînent une réduction du nombre de têtes de bétail de 10 à 50 p. 100 et de la production agricole de 2 à 10 p. 100 (18).

En effet, 60 p. 100 des bovins sont soumis au risque trypanosomien en zone subhumide en Afrique de l'Ouest (21). Leur contrôle est basé exclusivement sur l'utilisation de trypanocides curatifs ou préventifs, associée à un risque important de chimiorésistance. La lutte contre les glossines est considérée comme un bien public local. Aucune technique communautaire de lutte anti-vectorielle qui n'est 
pas basée sur les traitements individuels des animaux n'est donc adoptée par les éleveurs, en dehors des projets de développement ou de recherche (20).

Dans cette même zone géographique, $A$. variegatum est la tique la plus nuisible (31). Les tiques sont reconnues pathogènes et contrôlées de manière individuelle par arrachage manuel, pulvérisation d'insecticide et pour on, techniques coûteuses en temps pour la première, économiquement pour les deux autres $(7,32,33)$.

Au Burkina Faso, la seule technique de lutte conjointe contre les tiques et les glossines financée par les éleveurs est basée sur l'usage de pour on de fluméthrine (4) ; or cette technique reste coûteuse et inaccessible pour les éleveurs traditionnels. L'amélioration des conditions sanitaires de production et l'intensification nécessaire entraînent donc le besoin d'innovation.

L'étude de l'écologie comportementale d'A. variegatum (en particulier son mode d'invasion par une fixation temporaire entre les onglons) a permis de mettre au point une méthode innovante contre cette espèce, le pédiluve acaricide (30), qui s'est par la suite avérée efficace contre les tsé-tsé. Le traitement régulier des animaux par pédiluve permet alors de réduire de plus de 90 p. 100 l'incidence trypanosomienne dans certaines conditions $(8,9)$. Cette méthode est efficace, rapide et économique mais repose sur l'application de recommandations techniques strictes. Elle correspond à une méthode de protection individuelle contre les tiques, mais collective contre les glossines (10).

Cette innovation a été élaborée à partir de tests en station et en milieu paysan durant sept ans auxquels ont succédé une phase de vulgarisation à partir de fiches techniques, l'invitation des élus à des ateliers de présentation de la méthode avec visite sur le terrain, l'animation de débats au sein des organisations d'éleveurs, la facilitation des échanges de paysan à paysan et l'élaboration de cahiers des charges. Cette étude a été motivée par le besoin de connaître l'importance de l'adoption de l'innovation (l'appropriation de cette invention par les éleveurs au Burkina Faso) ainsi que les facteurs d'adoption. En effet, « une technique adaptée n'est pas forcément adoptée » (22). De manière subsidiaire, il était intéressant de savoir quelles étaient les modalités d'usage de la méthode, en particulier quels étaient les écarts au protocole observés.

\section{MATERIEL ET METHODES}

\section{Cadre conceptuel : l'innovation}

Héritier (2001) a écrit : «L'invention n'est pas l'innovation, même si elle en est la condition » (16). L'innovation a été initialement étudiée dans le domaine industriel. Ainsi Schumpeter (29) définissait l'innovation comme la combinaison de facteurs de production qui peut s'exprimer par la fabrication d'un nouveau produit, une nouvelle manière de produire, la mise en place de nouveaux débouchés, ou l'accès à de nouvelles ressources.

Dans le cas présent, il s'agit d'une innovation qui apporte une nouvelle manière de produire, grâce à une nouvelle méthode de lutte sanitaire. C'est un nouveau service qui est proposé au groupe ou un outil individuel selon le cas. On peut parler d'innovation modificative car l'organisation du travail va changer (fréquence et temps de traitement, ressources humaines mobilisées). L'éleveur dispose d'un nouveau service de lutte sanitaire qui doit permettre de produire plus sur les mêmes unités de surface agricole grâce à l'amélioration des conditions sanitaires et il est aussi attendu que cette méthode allège la quantité de travail (nombre de personnes mobilisées et temps passé pour le traitement) et donc s'insère dans une démarche d'intensification.
Une invention devient innovation lorsqu'elle est adoptée par le corps social (à l'échelle individuelle, on parle seulement de changement de pratique), et elle existe alors grâce à un réseau sociotechnique composé de producteurs, de chercheurs, de techniciens... Une innovation est un processus dynamique (tourbillonnaire) et créatif par lequel un groupe social s'approprie une nouveauté et la co-construit dans le temps en tenant compte de différentes dimensions (techniques, culturelles, économiques, organisationnelles) et des savoir-faire locaux (11).

Dans la recherche-développement, les chercheurs considèrent que les améliorations organisationnelles favorisent l'adoption des améliorations techniques. Jouve a émis l'hypothèse que certaines innovations techniques ne sont pas adoptées par les producteurs en dépit de leur efficacité technique du fait que les aspects sociaux et économiques ne sont pas pris en compte (19). Non seulement toute innovation entraîne des changements sociaux sous l'effet de l'action du réseau socio-technique, mais en plus, dans le cas d'un bien collectif, les difficultés de la gestion collective d'un outil se posent. Or, dans le contexte de l'élevage au Burkina Faso (20), comme dans la majorité des pays africains (15), les éleveurs montrent une préférence nette pour les méthodes de lutte sanitaire individuelles et le prêt est la pratique de gestion la plus fréquente pour le matériel utilisé par plusieurs personnes. Une modification des pouvoirs peut se produire par émergence de nouveaux leaders qui bousculent alors l'autorité traditionnelle et renforcent certaines inégalités sociales ou en créent de nouvelles. Des conflits sociaux peuvent être exacerbés, notamment sur la gestion de l'espace et de l'accès aux ressources naturelles (1).

Cinq critères d'évaluation de l'adoptabilité des innovations ont été proposés par Mendras et Forsé (23) : l'avantage relatif apporté par l'innovation par rapport à la situation initiale, sa compatibilité par rapport au système en place, sa plus ou moins grande complexité, son « essayabilité » dans le contexte du producteur, son observation chez autrui. En effet, il s'agit d'être le plus proche possible de l'évaluation, réalisée par le producteur, du bénéfice par rapport au risque (28). Cette évaluation est complexe et dépend en partie de l'expérience et des savoirs des paysans. Ces éléments sont souvent insuffisamment connus dans le contexte africain. La diversité des conditions de production (climat, écologie, géographie, sociologie) entraîne une limitation des conditions de validité des innovations. Alary (1) a constaté que «les petits paysans ou petits éleveurs des pays en développement sont souvent réticents face aux innovations technologiques issues de la recherche ». En effet, les producteurs travaillant dans des conditions difficiles et dont la survie économique est inféodée aux aléas (climatiques, économiques...) ont une attitude conservatrice et recherchent avant tout à prendre le moins de risques possible pouvant menacer soit leurs revenus et la survie de l'exploitation familiale, soit le système social au sein duquel ils ont établi des liens de solidarité et des liens professionnels (entre acteurs de la filière) (1). Ainsi les réticences des producteurs à adopter les mesures d'intensification préconisées en particulier par la recherche sont liées à «l'accroissement de la vulnérabilité actuelle à l'égard du marché » (1).

\section{Zone d'étude}

L'étude a été conduite au Burkina Faso en Afrique de l'Ouest dans les zones périurbaines de la capitale, Ouagadougou, et de la deuxième ville du pays, Bobo-Dioulasso. Le climat est soudano-sahélien à Ouagadougou et soudanien à soudano-guinéen à Bobo-Dioulasso (respectivement 700 et $1050 \mathrm{~mm}$ de pluviométrie annuelle) (2). Les tiques A. variegatum sont présentes dans ces deux zones et représentent une contrainte majeure à l'élevage de bovins (30). L'anthropisation importante de la périphérie de la capitale a pour 
conséquence une dégradation importante de la végétation naturelle et des cordons ripicoles, d'où une disparition des glossines riveraines (13). La majorité des élevages sédentaires de cette zone ne sont donc pas soumis au risque trypanosomien. En revanche, à la périphérie de Bobo-Dioulasso, située dans le bassin du Mouhoun, le risque est élevé (5).

Les élevages les plus modernes du pays, en relation avec les contraintes sanitaires et la proximité d'un marché de consommation plus important, sont situés à la périphérie de Ouagadougou. Les élevages de l'Association des promoteurs de lait local du Kadiogo (APLL), étudiés ici, font partie de ces élevages laitiers sédentaires et intensifs. A la périphérie de Bobo-Dioulasso, on trouve généralement des élevages transhumants, utilisant des races locales et à faible utilisation d'intrants, de type extensif (92 p. 100 des élevages périurbains de la périphérie de Bobo-Dioulasso) (14). Certains élevages sont entrés dans une démarche d'intensification.

L'étude ayant été conduite en 2008, les pédiluves étudiés ont été ceux mis en place avant 2007, car il n'était pas possible d'analyser correctement l'adoption sur la première année de mise en place. Tous les pédiluves ont été identifiés et géoréférencés (figure 1). Les éleveurs devant faire l'objet de l'enquête étaient tous les bénéficiaires attendus, c'est-à-dire ceux qui avaient demandé leur mise en place et ceux qui étaient membres de l'Organisation professionnelle d'éleveurs (OPE) et qui se trouvaient à moins de deux kilomètres dans le cas des pédiluves collectifs, ainsi que les nonmembres à qui le service avait été proposé. En tout, 22 pédiluves ont été étudiés et 72 éleveurs ont été interrogés.

Trois types de questionnaires ont été remplis : un questionnaire « vie associative », un questionnaire « gestion technique et financière du pédiluve » et un questionnaire « éleveur». Le questionnaire « vie associative » a été soumis aux élus de chaque OPE où au moins un pédiluve a été mis en place (de manière individuelle ou collective). Tous les parcs de nuit des éleveurs interrogés ont été géoréférencés. Le questionnaire " gestion technique et financière du pédiluve » a été soumis à au moins un des gérants du pédiluve, avec l'éleveur concerné s'il s'agissait d'un pédiluve individuel ou avec au moins deux élus de l'OPE s'il s'agissait d'un pédiluve collectif. Le questionnaire «éleveur » a été soumis à chaque éleveur de manière individuelle.

\section{Traitement statistique des données}

Les données de l'enquête ont été gérées par une base de données relationnelle sous Access. Un premier dépouillement des questionnaires a permis de trier les variables et d'éliminer celles qui présentaient des réponses très peu variables (moins de 5 p. 100 de variabilité) au sein de la population étudiée. Les variables pour lesquelles les auteurs n'ont pas pu obtenir de réponses fiables pour la totalité des personnes enquêtées ont été éliminées. Parmi les variables liées entre elles, par exemple celles qui décrivaient le système de production, ont été gardées celles qui étaient les plus représentatives des pratiques d'élevage pouvant influencer l'adoption. Suite à des analyses préliminaires, les auteurs ont ainsi gardé 21 variables considérées comme " actives » pour décrire les pratiques et perceptions des éleveurs, dans le souci de conserver un ratio lignes/ colonnes proche de 5 (figure 2). Les données quantitatives (six variables) ont été codées en classes (par l'utilisation des quartiles) pour réaliser une analyse des correspondances multiples (ACM) afin de décrire les corrélations entre variables et de caractériser les éleveurs étudiés (34). Une classification hiérarchique ascendante a ensuite été réalisée pour calculer des distances entre éleveurs (méthode de Ward), à partir de leurs coordonnées sur les quatre premiers axes principaux. Trois groupes d'éleveurs se sont alors distingués (figure 3) et ont été projetés sur le premier plan de l'ACM.
Les 21 variables actives sont décrites ci-dessous (tableau I). Les variables correspondant aux connaissances des éleveurs dans le domaine sanitaire, et en particulier celles portant sur les insectes et les acariens n'ont pas été retenues car les analyses préliminaires ont montré qu'elles expliquaient de manière très faible l'inertie du nuage de points.

Pour chaque variable a été calculée une valeur correspondant à l'inertie cumulée de celle-ci sur les quatre axes en additionnant les valeurs absolues des contributions de chaque modalité (en appliquant à chaque axe un coefficient proportionnel à sa contribution à l'inertie). Les dix premières variables actives ont alors été retenues pour décrire les groupes d'éleveurs (figures 2 et 3). La corrélation entre variables a été étudiée par la méthode du cercle des corrélations.

Afin de décrire l'adoption de la technique, sept variables ont été retenues comme des indicateurs d'adoption : l'utilisation individuelle du pédiluve, correspondant au nombre de saisons des pluies pendant lesquelles le troupeau a utilisé le pédiluve, le ratio entre la durée d'utilisation individuelle et le nombre d'années d'existence du pédiluve, le ratio entre le nombre de troupeaux utilisateurs réels et potentiels, le ratio entre le nombre de bovins traités et susceptibles de l'être, la fréquence de passage au mois de juin de la dernière année d'usage, le nombre de mois d'usage par année et enfin le nombre total de passages la dernière année d'usage. Les troupeaux utilisateurs potentiels ont été les troupeaux des éleveurs membres d'une OPE au sein de laquelle un pédiluve collectif a été

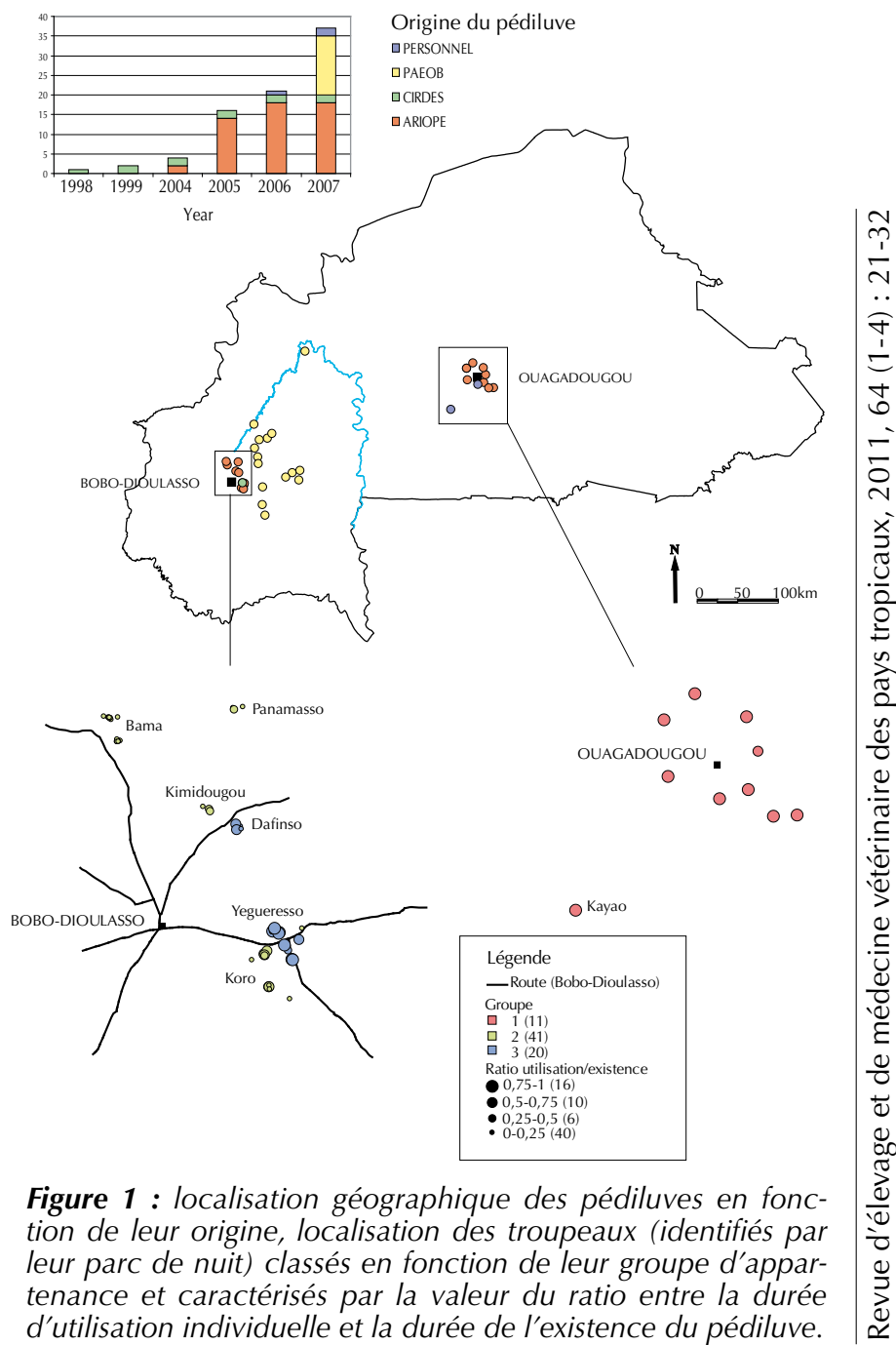




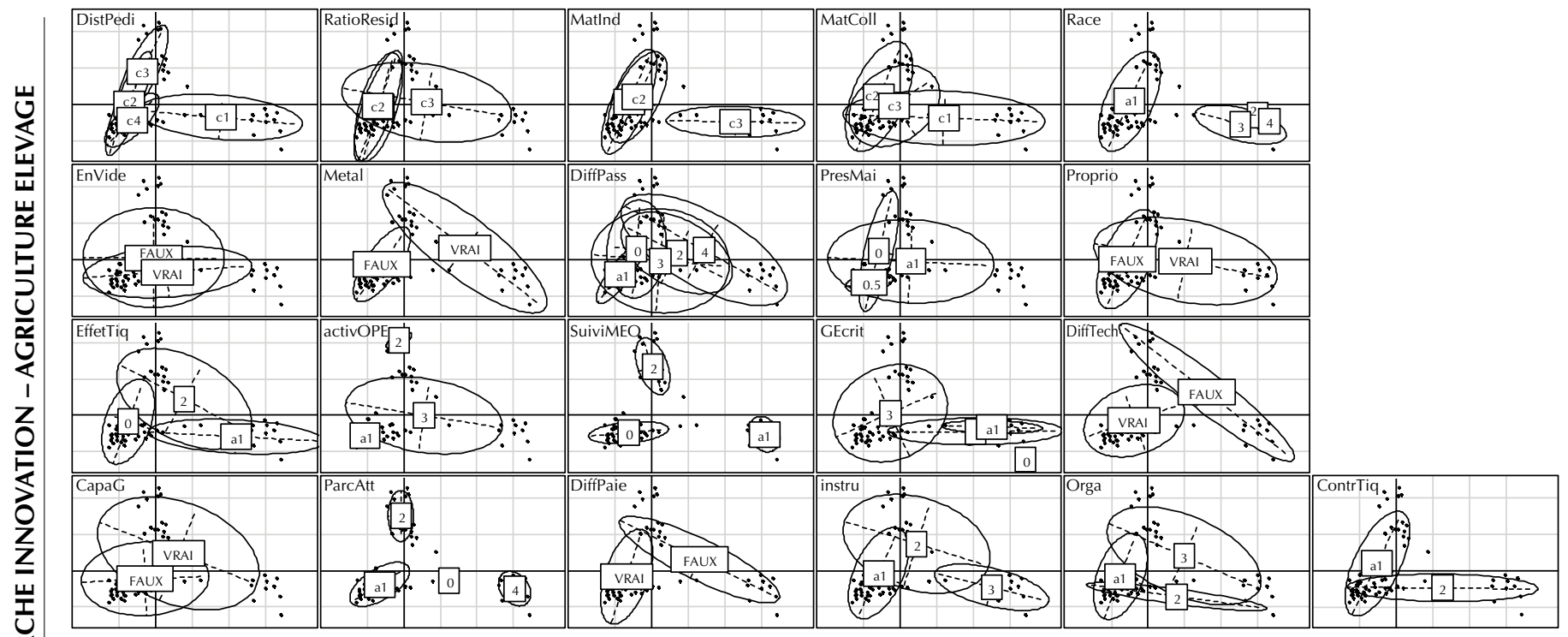

Figure 2 : projection des différentes modalités des variables descriptives sur le premier plan de l'ACM.

DistPedi : distance en mètres entre le parc de nuit et le pédiluve (à partir des coordonnées GPS), transformée en classes avec les quartiles (c1 de 0 à $209 \mathrm{~m}$; c2 de 210 à $427 \mathrm{~m}$; c3 de 427 à 1187,5 m;c4 > 1187,5 m).

RatioResid : ratio entre le nombre de bovins déclarés restant au campement toute l'année et le nombre total de bovins déclarés dans le troupeau), transformé en classes avec les quartiles $(c 1 \leq 0,1 ; 0,1<c 2<1 ; c 3=1)$.

Matlnd : nombre de catégories de matériel ou d'infrastructures agricoles utilisées de manière individuelle par l'éleveur, transformé en classes $(\mathrm{c} 1 \leq 1 ; 2 \leq \mathrm{c} 2 \leq 3 ; \mathrm{c} 3>3)$.

MatColl : nombre de catégories de matériel ou d'infrastructures agricoles utilisées de manière collective par l'éleveur, transformé en classes $(\mathrm{c} 1=0 ; \mathrm{c} 2=1 ; \mathrm{c} 3 \geq 2)$.

Race : la majorité des bovins sont des zébus Peuhls (métissés ou non avec des taurins) (a1), de race locale améliorée (GoudhaliAzawakh ou métissés avec race européenne) avec dominance de race locale (a 2), la majorité sont des métis avec une dominance de sang exotique européenne (a3), race exotique européenne pure (a4).

EnVide : le troupeau a été entraîné à passer dans le pédiluve à vide : VRAI; sinon : FAUX.

Metal : le troupeau utilise un parc de vaccination ou une stabulation en métal : VRAI ; sinon : FAUX.

DiffPass : aucune difficulté de passage pour le troupeau (a4), difficultés surmontées (a3), difficultés d'une partie du troupeau persistantes (a2), difficultés de l'ensemble du troupeau persistantes (a1).

PresMai : le troupeau est présent au campement au mois de mai (a1), il revient au mois de mai (a0,5), est absent (a0).

Proprio : I'éleveur est propriétaire des terres agricoles : VRAI ; sinon : FAUX.

EffetTiq : effet du pédiluve sur les tiques observé par l'éleveur : pas d'effet observé (a0), effet partiel contre les tiques (a1), bonne efficacité contre les tiques (a2).

activOPE : des activités avec gestion financière ont été menées au sein de l'OPE (a3), des activités sans gestion financière ont été menées (a2), l'OPE n'a exercé qu'une fonction de représentation (a1).

SuiviMEO : les éleveurs ont été suivis régulièrement lors de la mise en œuvre des services : par la recherche (a2), par un technicien ou assimilé (a1), pas de suivi régulier après la mise en place (a0).

GEcrit : le gérant sait écrire et lire (a3), il est aidé par une personne alphabétisée (a2), le gérant ne sait ni lire ni écrire, et il n'est pas aidé (a1).

DiffTech : il existe des difficultés techniques pour la gestion du pédiluve (maîtrise du dosage, difficultés à faire passer les animaux...) : VRAl ; il n'y a pas de difficultés techniques : FAUX.

CapaG : le gérant est capable de remplir ses fonctions : VRAI (sait doser le produit) ; sinon : FAUX.

ParcAtt : le parc d'attente correspond à la stabulation (a4), ils est rond avec du grillage (a1), traditionnel et en forme d'entonnoir (a3), intermédiaire (parc avec grillage et forme d'entonnoir ou en branchages mais rond) (a2), absence (aO).

DiffPaie : il y a des difficultés de paiements : VRAI ; sinon : FAUX.

Instru : I'éleveur a reçu une éducation uniquement traditionnelle (a1), il est allé à l'école élémentaire (a2), dans un établissement du secondaire (a3).

Orga : selon l'éleveur, la mise en œuvre de la lutte par pédiluve est aisée et pratique au niveau de l'organisation du travail (a3), ou contraignante, difficile (a2) ou n'a pu être appréciée (pas d'usage = a0).

ContrTiq : au niveau de l'OPE, les tiques sont la contrainte citée en premier (a4), en deuxième (a3), en troisième (a2) ou ne figurent pas parmi les trois premières contraintes (a1).

construit et dont le parc de nuit était installé à moins de deux kilomètres, ainsi que les troupeaux des éleveurs non-membres qui ont utilisé le pédiluve. Les bovins susceptibles d'être traités étaient les bovins appartenant à ces troupeaux. Ces variables, toutes quantitatives, ont été soumises à une analyse en composantes principales (ACP). Les trois groupes d'éleveurs caractérisés par leurs pratiques ont été projetés sur le premier plan de l'ACP pour comparer leur niveau d'adoption. L'adoption a ensuite été comparée entre les groupes pour chaque indicateur. Pour cela, la normalité des distributions intra-groupes a été vérifiée par un test de KolmogorovSmirnov (12). Ces distributions n'étant pas normales, l'effet global du groupe a été testé par une analyse Anova de Kruskal et Wallis (17). Lorsqu'il a été significatif, les groupes ont alors été comparés par un test non paramétrique de comparaisons multiples de Steel (26). Toutes les analyses on été réalisées avec le logiciel en accès libre R 2.9.2 (27). 


\section{Tableau I}

Classement des 21 variables actives

\begin{tabular}{|c|c|c|c|}
\hline $\begin{array}{l}\text { Catégorie des } \\
\text { variables actives }\end{array}$ & $\begin{array}{l}\text { Modalité de mise en œuvre } \\
\text { du service }\end{array}$ & Typologie d'élevage & Perception \\
\hline $\begin{array}{l}10 \text { variables } \\
\text { principales }\end{array}$ & $\begin{array}{l}\text { Type du parc d'attente }{ }^{1} \\
\text { Type de suivi de la mise en place } \\
\text { Difficultés de paiement }\end{array}$ & $\begin{array}{l}\text { Race des bovins } \\
\text { Usage de parc métallique } \\
\text { Instruction de l'éleveur } \\
\text { Quantité de matériel individuel utilisé } \\
\text { Type d'activités menées par l'OPE }{ }^{2}\end{array}$ & $\begin{array}{l}\text { Efficacité du pédiluve } \\
\text { sur les tiques } \\
\text { Facilité de mise en } \\
\text { œuvre }\end{array}$ \\
\hline $\begin{array}{l}11 \text { variables } \\
\text { secondaires }\end{array}$ & $\begin{array}{l}\text { Distance pédiluve parc de nuit } \\
\text { Difficultés techniques } \\
\text { Alphabétisation du gérant } \\
\text { Difficultés de passage } \\
\text { Réalisation de l'entraînement du } \\
\text { passage à vide } \\
\text { Capacités du gérant }\end{array}$ & $\begin{array}{l}\text { Importance des tiques comme contrainte } \\
\text { Propriété des terres } \\
\text { Quantité de matériel collectif utilisé } \\
\text { Ratio de bovins résidents } \\
\text { Présence des troupeaux au mois de mai }\end{array}$ & \\
\hline
\end{tabular}

${ }^{1}$ Le parc d'attente est l'enclos précédent le pédiluve où le troupeau entier entre et est contenu le temps de faire passer les animaux un à un dans le pédiluve.

${ }^{2}$ Organisation professionnelle d'éleveurs

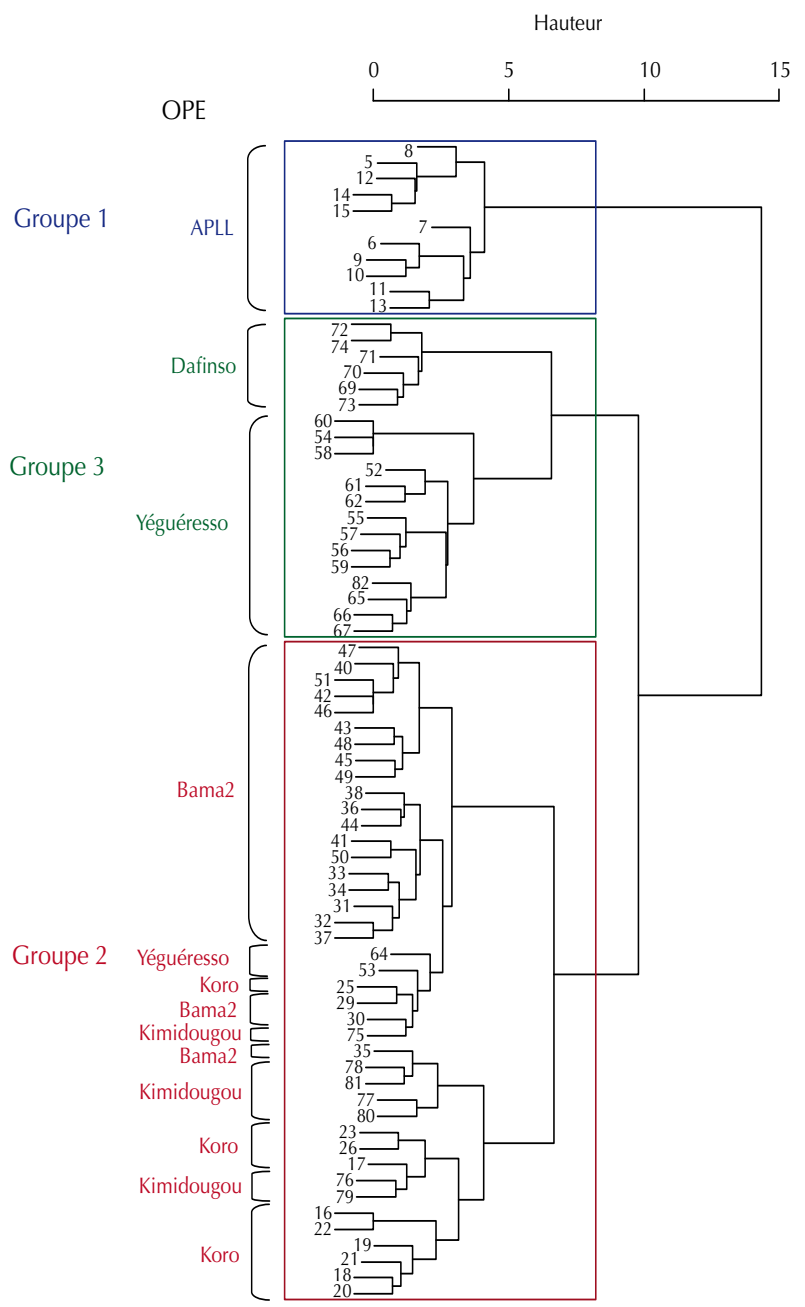

Figure 3 : identification de trois groupes d'éleveurs bien différentiés en fonction de leurs pratiques et de leurs perceptions par classification hiérarchique ascendante (méthode de Ward). OPE : Organisation professionnelle d'éleveurs.

\section{RESULTATS}

\section{Contribution des variables descriptives à l'inertie}

Les dix variables qui ont contribué le plus à l'inertie du nuage de points ont été, par ordre décroissant d'importance : la nature du parc d'attente, la nature de la mise en place du service, la race des bovins, l'utilisation d'un parc métallique, les difficultés de paiement, l'effet observé sur les tiques, le niveau d'instruction, la quantité de matériel individuel, le type d'activités menées par l'OPE et la facilité de mise en œuvre de la méthode (par rapport à l'organisation du travail). Leurs modalités se sont projetées de manière très distincte sur le premier plan de l'ACM (figure 2).

\section{Corrélations entre variables}

Sur le premier axe, le parc d'attente correspondant à la stabulation a été la modalité expliquant de la manière la plus importante l'inertie du nuage de points sur l'axe 1 de l'ACM : elle a été fortement corrélée à un niveau d'instruction élevé (secondaire et plus), à l'utilisation de races améliorées (métis avec dominance de race européenne et individus de race européenne non métissée), à une distance pédiluve parc d'attente très faible, ainsi qu'avec le suivi par un technicien, l'absence de matériel collectif, un large équipement individuel (plus de trois types de matériel individuel), la contrainte représentée par les tiques importante (troisième contrainte d'élevage) et un effet partiel observé sur les tiques ( $\mathrm{p}<0,05$; figure 3 ). L'utilisation de parc (attente ou stabulation) en métal a été associée à l'absence de difficultés de passage et à l'absence de difficultés de paiement $(\mathrm{p}<0,05)$ ainsi qu'à une appréciation positive de la facilité de mise en œuvre de la méthode. La modalité OPE avec activités sans gestion financière a été la modalité la plus importante sur le deuxième axe de l'ACM. Elle a été corrélée avec les parcs d'attente intermédiaires et le suivi de la mise en place par la recherche ainsi qu'avec une distance pédiluve parc de nuit importante d'environ un kilomètre (troisième quartile, entre 787 et $1188 \mathrm{~m})(\mathrm{p}<0,05)$. 


\section{Description des trois groupes par rapport aux variables importantes}

La projection des trois groupes sur le premier plan factoriel de l'ACM montre qu'ils étaient bien séparés par cette analyse de leurs caractéristiques (figure 4). Le premier axe sépare le groupe 3 des groupes 1 et 2, et le deuxième axe isole le groupe 1 par rapport aux groupes 2 et 3 . On observe que les individus d'une même OPE étaient proches dans la classification, ce qui était lié au fait que certaines variables ont été mesurées à l'échelle de l'OPE ou du pédiluve. Cependant, cela n'était pas systématique.

Le premier groupe d'éleveurs s'est distingué davantage des deux autres et a comporté 11 élevages : les dix éleveurs de Ouagadougou (dont l'un possédait deux élevages). Le deuxième groupe a comporté 41 individus : il a rassemblé l'intégralité des éleveurs interrogés de trois OPE : les groupements des villages de Koro, Bama 2 et Kimidougou. Deux éleveurs de Yegueresso en ont fait aussi partie. Le dernier groupe a rassemblé 20 individus : la majorité des éleveurs de l'OPE de Yegueresso et tous ceux de Dafinso (figure 1). La représentation des individus et des variables sur le premier plan factoriel de l'ACP a montré que le groupe 1 (éleveurs de Ouagadougou) était associé aux modalités qui décrivent l'élevage moderne. Ce groupe s'est distingué par une majorité de parcs d'attente représentés par la stabulation (91 p. 100) et un seul parc d'attente absent (soit 9 p. 100). Les animaux étaient complètement sédentaires (ils n'allaient pâturer qu'à proximité immédiate en saison des pluies). Le suivi par un technicien (82 p. 100) a été le type de suivi le plus fréquent alors que l'absence de suivi de la mise en place a concerné seulement 18 p. 100 de l'effectif. En effet, au sein de l'APLL, un éleveur, dont le niveau technique était élevé, a proposé un suivi des exploitations comme prestation technique. La race locale zébu Peuhl n'a été majoritaire que dans un seul élevage du groupe 1 (9 p. 100). La race la plus fréquente a été représentée par des métis avec des races européennes (45 p. 100). Des races européennes non métissées et des races locales améliorées ont aussi été rencontrées (par exemple, Goudhali, Azawakh). L'utilisation d'un parc métallique (stabulation ou parc de vaccination) a aussi illustré les différences de pratiques puisque la totalité du groupe 1 l'a utilisé contrairement aux autres groupes. Il n'y a pas eu de difficultés de paiement, ce qui était normal puisque les pédiluves étaient utilisés individuellement. La grande majorité (73 p. 100) des éleveurs avaient un niveau scolaire élevé, au moins celui du secondaire. Le niveau d'équipement individuel était caractéristique des élevages modernes : en effet, la totalité du groupe était très équipée (plus de trois catégories de matériel individuel utilisé). L'équipement en matériel collectif a été en revanche rare (82 p. 100 n'en possédaient pas). L'intégralité des éleveurs appartenaient à des OPE qui avaient des activités avec gestion financière. Une bonne efficacité du traitement a été observée par 55 p. 100 des individus, un effet partiel par un tiers d'entre eux et 9 p. 100 n'ont observé aucun

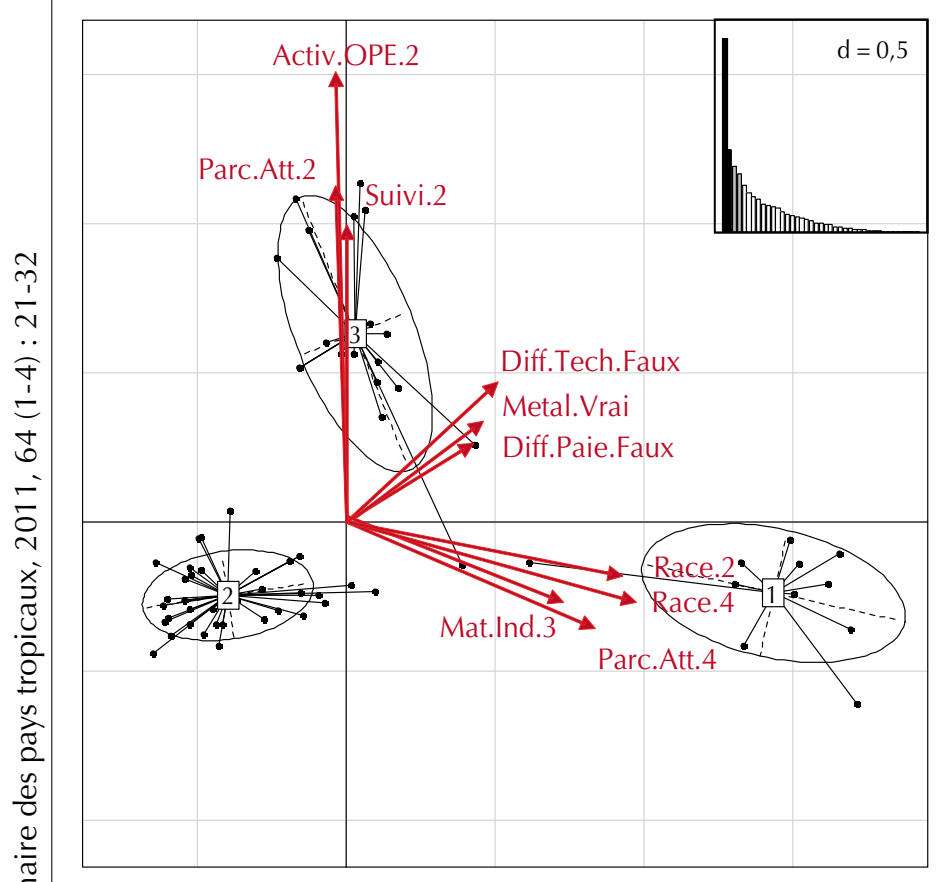

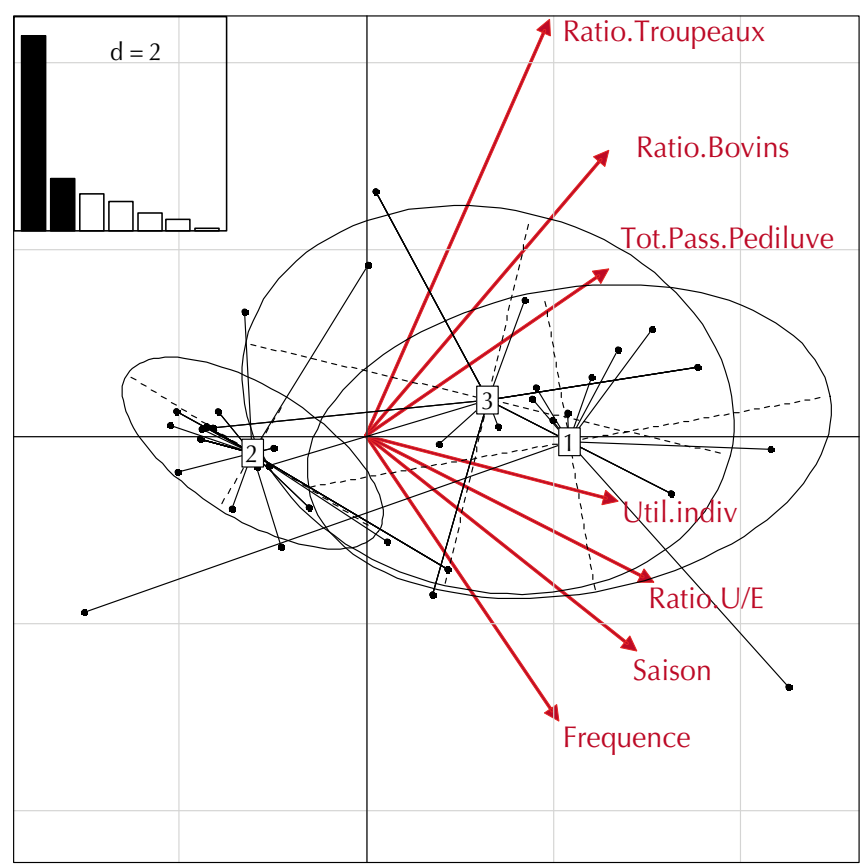

b

Figure 4 : projection des trois groupes d'éleveurs discriminés par la classification hiérarchique ascendante sur les premiers plans de l'analyse des correspondances multiples (réalisée sur les variables descriptives) et de l'analyse en composantes principales (réalisée sur les indicateurs d'adoption) de gauche à droite respectivement. Les modalités les plus importantes sont indiquées par des flèches rouges.

Activ.OPE.2 : absence d'activité avec gestion financière dans l'OPE ; Parc. Att.2 : usage d'un parc d'attente intermédiaire ; Suivi.2 : appui technique apporté par la recherche; Diff.Tech.Faux : absence de difficultés techniques ; Metal.Vrai : utilisation d'un parc métallique ; Diff.Paie.Faux : absence de problème de paiement ; Race. 2 : bovins métissés avec des races européennes majoritaires ; Race.4 : races exotiques européennes majoritaires ; Parc.Att.4 : le parc d'attente est constitué par la stabulation ; Mat.Ind.3 : utilisation d'au moins trois types de matériel individuel ; Ratio. Troupeaux : ratio du nombre de troupeaux traités sur ceux ayant accès au pédiluve ; Ratio.Bovins : ratio du nombre de bovins traités sur le nombre de bovins ayant accès au pédiluve ; Tot.Pass.Pediluve : nombre total de bovins passés dans le pédiluve pendant une saison de traitement; Util.indiv : nombre d'années d'utilisation pour l'éleveur ; RatioUE : ratio du nombre d'années d'utilisation individuelle sur la durée d'existence du pédiliuve ; Saison : durée de la saison de traitement (mois) par an ; Frequence : fréquence d'utilisation du pédiluve au mois de juin de la dernière année de traitement. 
effet du traitement sur les tiques. Une grande majorité d'éleveurs (73 p. 100) ont trouvé que le pédiluve était une méthode de lutte pratique et facile à mettre en œuvre.

Les groupes 2 et 3 appartenaient au même sous-ensemble d'éleveurs traditionnels de la périphérie de Bobo-Dioulasso - ils étaient membres de groupements de l'Union des éleveurs producteurs de lait du Houet (UEPL) - mais présentaient des caractéristiques différentes.

Le groupe 2 a rassemblé le plus grand nombre d'individus (41). La totalité des pédiluves avaient un parc d'attente rond avec du grillage. L'absence de suivi de la mise en place du service (après la construction) a concerné l'intégralité du groupe. Les zébus Peuhls (plus ou moins métissés avec des taurins trypanotolérants) appartenaient aux éleveurs de manière exclusive. Dans le groupe 2, l'utilisation d'un parc métallique (parc d'attente ou de vaccination) a concerné un seul individu (2,4 p. 100). Les difficultés de paiement ont été caractéristiques puisque 97,6 p. 100 des éleveurs ont présenté des difficultés pour payer le service. La plupart (93 p. 100) des éleveurs ont reçu uniquement une éducation traditionnelle. La majorité du groupe 2 (70 p. 100) avait un niveau d'équipement individuel très bas ( 0 ou 1 catégorie de matériel individuel possédé) et était plutôt équipé en matériel collectif (80 p. 100). La majorité des éleveurs (78 p. 100) n'ont pu donner leur avis sur l'aspect pratique de la méthode de lutte car l'usage était absent ou trop ponctuel. Dans le groupe 2, ont été observées uniquement des OPE qui n'ont assuré qu'un rôle de représentation, sans activités réelles (54 p. 100). Seulement 46 p. 100 des éleveurs appartenaient à des OPE qui avaient des activités avec gestion financière. Par ailleurs, 83 p. 100 des éleveurs n'ont observé aucun effet du traitement sur les tiques : ceci a été lié au fait que l'utilisation du pédiluve a été très faible dans ce groupe. Seulement 13 p. 100 des éleveurs ont observé une bonne efficacité contre les tiques. Un effet partiel a été observé chez 5 p. 100 des individus.

Le groupe 3 était un sous-ensemble de 20 individus. Il était majoritairement ( 75 p. 100 ) caractérisé par des parcs d'attente intermédiaires et seulement 25 p. 100 de parcs ronds avec grillage. Il faisait aussi l'objet d'un suivi par un centre de recherche (95 p. 100). En effet, les deux pédiluves mis en place par le Cirdes appartenaient à l'OPE largement représentée dans ce groupe (Yegueresso). De plus, le Cirdes a suivi pendant une saison des pluies l'utilisation du pédiluve dans le groupement de Dafinso pour mesurer l'efficacité de la méthode. L'absence de suivi de la mise en place a concerné seulement 5 p. 100 du groupe. La race majoritaire a été la race zébu Peuhl (95 p. 100), la possession de métis avec des races européennes est restée très discrète. Un tiers (35 p. 100) des éleveurs ont utilisé un parc métallique, représentant une valeur intermédiaire entre les groupes 1 et 2 . Les difficultés de paiement ont concerné un tiers (35 p. 100) du groupe, valeur à nouveau intermédiaire entre les groupes 1 et 2 . Les éleveurs ont reçu majoritairement une éducation traditionnelle (60 p. 100), mais 35 p. 100 d'entre eux sont allés à l'école élémentaire, ce qui est une valeur élevée par rapport au groupe 2. Comme le groupe 2, le groupe 3 avait un niveau d'équipement individuel très bas $(60 \mathrm{p} .100$ du groupe possédait 0 ou 1 catégorie de matériel individuel), mais 90 p. 100 des individus utilisaient du matériel collectif. Parmi les éleveurs, 65 p. 100 ont trouvé que le pédiluve était pratique à utiliser. La majorité (70 p. 100) des éleveurs appartenaient à des OPE qui avaient des activités avec gestion financière. Les individus ont observé le plus souvent (65 p. 100) une bonne efficacité du pédiluve contre les tiques alors que 35 p. 100 n'ont observé aucun effet du traitement.

\section{Localisation spatiale des éleveurs}

La localisation spatiale des éleveurs selon leur groupe et l'importance de l'utilisation individuelle par rapport à la durée d'existence de chaque pédiluve (ratio U/E) (figure 1) est intéressante à analyser au niveau des éleveurs de Bobo-Dioulasso (les éleveurs de Ouagadougou appartenaient tous au groupe 1). En effet, les éleveurs des OPE de Yegueresso et de Dafinso se sont distingués par leur appartenance au groupe 3 et par leurs ratios U/E plus élevés. Or, le Cirdes est intervenu de manière conséquente dans ces deux OPE : les deux premiers pédiluves expérimentaux ont été construits à Borodougou et Tondogosso au bénéfice d'éleveurs appartenant à cette OPE et le suivi technique a été réalisé sur de nombreuses années (environ sept ans). Il est remarquable d'observer qu'en 2007 c'est dans cette OPE que le plus grand nombre de pédiluves a été construit (4). A Dafinso, un suivi de l'efficacité du pédiluve a été réalisé pendant la saison des pluies 2007 ; la présence régulière de l'équipe du Cirdes a représenté un réel appui. Les éleveurs du groupe 2 étaient plus éloignés de Bobo-Dioulasso ainsi que des routes goudronnées.

\section{Description de l'adoption dans les trois groupes}

La projection des groupes sur le premier plan factoriel de l'ACP (figure 4) montre que le groupe 2 s'est distingué des deux autres et que le recouvrement des groupes 1 et 3 a été important. En effet, les groupes 1 et 3 étaient ceux qui avaient adopté la méthode : les valeurs prises par leurs indicateurs d'adoption ont été proches. Le groupe 2 est le groupe qui ne l'a pas adopté. Le premier plan factoriel de l'ACP montre que le premier axe (qui représente 56 p. 100 de l'inertie globale) séparait le groupe 2 des groupes 1 et 3 . Le recouvrement des groupes 1 et 3 indique que ceux-ci ont eu des niveaux d'adoption proches, bien que représentant des systèmes d'élevage très différents.

Les profils d'adoption (figure 5), bien que cohérents par groupe, ont été différents d'un indicateur à l'autre, ce qui indiquait que l'information apportée par chacun d'eux était différente. Cela a été confirmé par l'absence de corrélation entre ces variables ( $\mathrm{p}>0,05)$.

Tous les indicateurs d'adoption étaient construits de manière à augmenter avec l'intensité de l'adoption.

Pour le groupe 2, les valeurs prises par les indicateurs ont toujours été très faibles et inférieures à celles des deux autres groupes $(\mathrm{p}<0,05)$. Dans ce groupe, des médianes nulles ont été observées pour le nombre total de passages, le ratio bovins traités sur bovins candidats, la fréquence mensuelle de passage et la durée d'utilisation annuelle. L'adoption n'a donc pas été effective pour ce groupe. L'analyse de la durée d'utilisation individuelle a exprimé assez bien le défaut d'adoption dans le groupe 2 puisque la médiane s'est située à 0,5 saison des pluies, ce qui correspondait à des essais ponctuels la première année d'utilisation (l'usage est inférieur à une saison complète) ; de plus, la répartition était agrégée autour de cette valeur, ce qui signait aussi l'échec d'adoption. La médiane du nombre total de passages s'est située à 0 , ce qui était éloquent. Le troisième quartile a atteint 1500 passages ; il y a donc eu une certaine variabilité résiduelle. Le ratio durée de l'utilisation sur durée d'existence du pédiluve a présenté des valeurs faibles dans le groupe 2 par rapport aux autres groupes : la médiane a été à 0,2 contre 0,7 pour le groupe 3 et 1 pour le groupe 1. Le ratio troupeaux traités sur troupeaux candidats a été moins éloquent. Ces chiffres indiquent que les éleveurs de ce groupe n'ont pas du tout utilisé leur pédiluve ou l'ont utilisé de manière discrète, en faisant des essais ponctuels de passage, sans que cet usage ne devienne une pratique. 

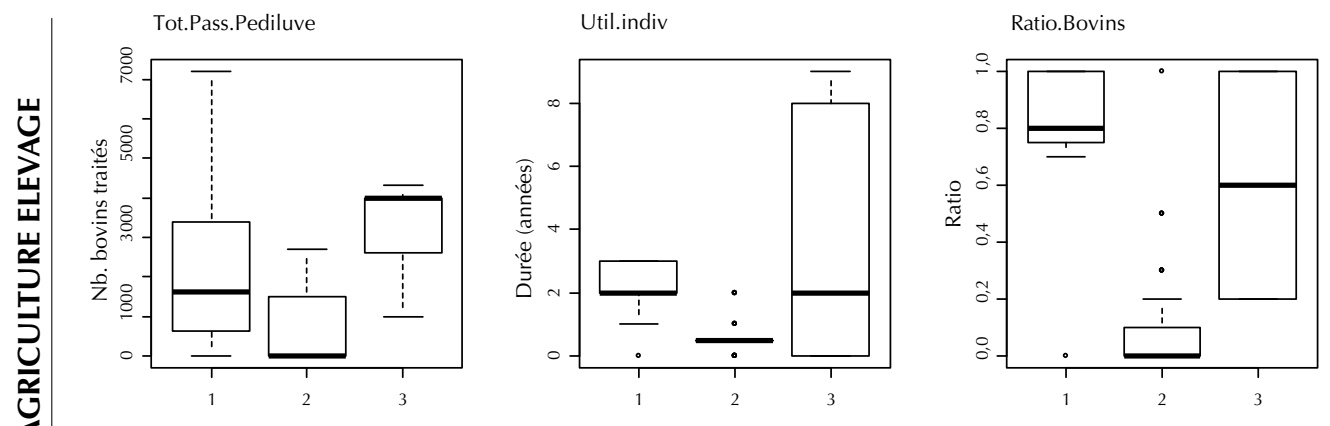

Ratio.Troupeaux

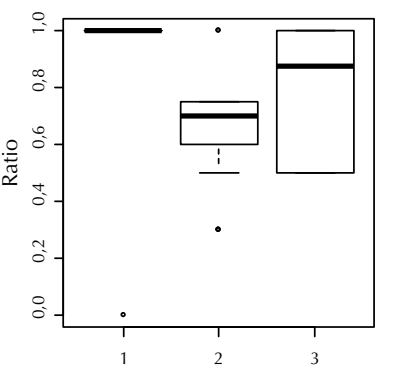

Frequence

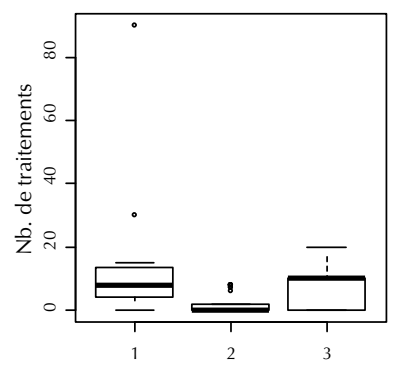

Saison

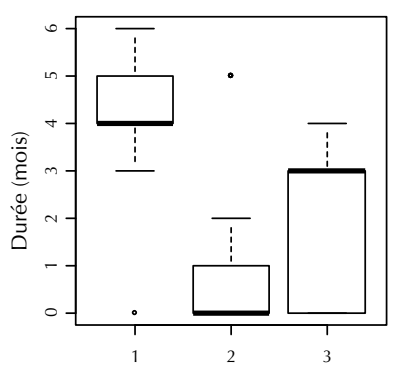

Ratio.U/E

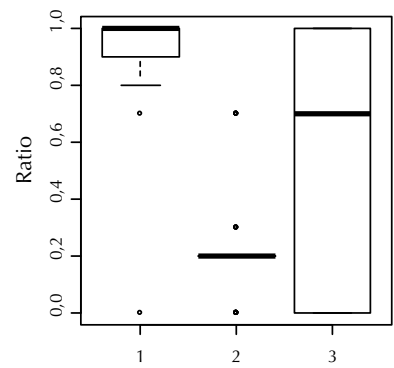

Tot.Pass.Pediluve : nombre total de bovins passés dans le pédiluve pendant une saison de traitement

Util.indiv : nombre d'années d'utilisation individuelle

Ratio.Bovins : ratio du nombre de bovins traités sur le nombre de bovins ayant accès au pédiluve

Ratio.U/E : ratio du nombre d'années d'utilisation individuelle sur la durée d'existence du pédiluve Ratio.Troupeaux : ratio du nombre de troupeaux traités sur ceux ayant accès au pédiluve

Frequence : fréquence d'utilisation du pédiluve pendant la dernière saison (juin) Saison : durée (mois) de la période de traitement pendant la dernière année d'utilisation

Figure 5 : boites à moustaches présentant les distributions simplifiées (quartiles, médianes, intervalles à 95 p. 100) des indicateurs d'adoption au sein des trois groupes.

La médiane du nombre total de passages par pédiluve lors de la dernière saison d'utilisation a été plus élevée dans le groupe 3 (égale à 4 000) mais le maximum le plus élevé a été atteint par le groupe 1 , chez qui les variations ont été les plus grandes. La moyenne des passages du groupe 3 a été égale à 3494 et celle du groupe 1 à 2377 (différence non significative $p=0,07$ ). Cet indicateur a mesuré le volume d'utilisation à l'échelle du pédiluve et n'a pas reflété les utilisations individuelles. Des résultats plutôt meilleurs ont été obtenus pour le groupe 3 car il s'agissait du groupe d'éleveurs traditionnels qui avaient un usage collectif de leurs pédiluves alors que les éleveurs modernes de Ouagadougou (groupe 1) avaient un usage individuel des pédiluves. Le nombre d'animaux ayant eu accès au pédiluve a donc généralement été supérieur dans le groupe 3 .

Concernant les groupes 1 et 3 , les médianes de la durée d'utilisation individuelle (nombre de saisons des pluies) ont été identiques et égales à deux ans mais les variations ont été très importantes dans le groupe 3 , avec notamment un maximum très élevé (égal à 9). Cependant, les durées moyennes n'ont pas été statistiquement différentes $(\mathrm{p}=0,83)$. La plus forte variabilité dans le groupe 3 s'est expliquée en grande partie par le fait que les deux premiers pédiluves ont été construits par la recherche il y a dix ans.

Le ratio utilisation individuelle sur durée d'existence du pédiluve a permis d'évaluer de quelle manière chaque éleveur a utilisé le pédiluve par rapport à la durée maximale possible d'utilisation. Ainsi cet indicateur a atteint des valeurs élevées pour le groupe 1 puisque la médiane a été égale à 1 , le premier quartile proche de 0,90 et la moyenne égale à 0,86 . Le groupe 3 a eu une médiane située à 0,70 , une moyenne égale à 0,53 , donc intermédiaire entre les deux autres groupes et une très forte variabilité (maximale) de ce paramètre a été observée. Les différences ont été significatives entre les groupes 1 et $3(p=0,04)$. Les durées d'utilisation des pédiluves par les éleveurs traditionnels n'ont pas été maximales alors que les éleveurs modernes ont utilisé leur pédiluve de manière maximale par rapport à la durée d'existence du pédiluve.
Dans leur cas, les durées d'utilisation individuelle ne pouvaient pas être meilleures (sauf pour deux individus), c'est donc l'existence récente des pédiluves qui a limité leurs scores.

$\mathrm{Au}$ niveau du ratio entre les bovins traités et les bovins candidats potentiels au traitement, une médiane élevée, égale à 0,8 , et une variabilité fiable pour le groupe 1 ont été observées. Dans le groupe 3, la médiane a été plus basse, égale à 0,6 , et la variabilité a été forte. Il n'y pas eu de différence significative entre ces deux groupes $(\mathrm{p}=0,45)$.

Le ratio troupeaux traités par rapport aux troupeaux candidats au traitement a donné des résultats différents. En effet, pour le groupe 1, il a pris la valeur 1 sauf pour un individu qui a présenté la valeur 0 (moyenne égale à 0,9 ). Cela signifiait que tous les pédiluves de ce groupe ont été utilisés de manière individuelle comme prévu et qu'il existait un pédiluve non utilisé. Pour le groupe 3, la médiane a été élevée, égale à 0,9 avec une variabilité assez importante (premier quartile égal à 0,5 ) et une moyenne égale à 0,8 . La différence entre les groupes 1 et 3 a été non significative $(\mathrm{p}=0,067)$.

Les fréquences mensuelles de passage ont été proches avec des médianes égales à 10 et 12 pour les groupes 1 et 3 , ce qui correspondait aux recommandations techniques d'un passage tous les deux à trois jours. Cependant deux valeurs ectopiques (30 et 90) ont été observées pour le groupe 1 qui représentaient des écarts au protocole importants (trois traitements par jour !). Les moyennes des fréquences ont été 17 et 78,1 respectivement pour les groupes 1 et 3 , mais cette différence n'a pas été significative $(p=0,99)$.

Enfin, le nombre de mois d'utilisation a été compris entre 3 et 6 mois avec une médiane et une moyenne égales à 4 mois pour le groupe 1 , contre une utilisation comprise entre 0 et 4 mois avec une médiane à 3 mois et une moyenne à 2,2 mois pour le groupe 3. Le groupe 3 a utilisé moins longtemps le pédiluve à chaque saison $(\mathrm{p}=0,002)$, ce qui correspondait à son caractère transhumant. 


\section{DISCUSSION}

\section{Pratiques, savoirs et adoption}

Alors que les savoirs paysans et les conceptions culturelles sont reconnus être fondamentaux pour expliquer les pratiques et leurs évolutions ("l'innovation relève profondément de l'acceptation socio-culturelle »;16), ici les connaissances concernant les maladies vectorielles et les vecteurs (tiques et glossines) se sont avérées faibles de manière homogène (données non présentées). Cependant, le niveau d'instruction a figuré parmi les dix plus importantes variables. Certes, plus le niveau d'instruction scolaire était élevé, plus l'individu concerné était sensible aux concepts scientifiques et aux pratiques modernes d'élevages. Mais cette variable a été en partie liée au système d'élevage car les doubles actifs avaient plus de moyens pour investir et leur double activité les autorisait à une prise de risque plus importante dans leur activité d'élevage qui ne leur assurait pas de manière exclusive leurs revenus. De plus, les personnes qui résidaient loin des centres urbains et des voies de communication étaient dans des systèmes sociaux plus traditionnels où les enfants allaient moins à l'école, et les réseaux sociotechniques dans lesquels ils se trouvaient étaient différents de ceux des personnes proches des voies de communication. En effet, si un centre de recherche a le choix entre deux zones d'étude équivalentes, il a tendance à choisir la zone la plus accessible pour des raisons pratiques. De plus, dans le cas de l'UEPL, toutes les réunions (conseil de gestion, ateliers, etc.) ont eu lieu à Bobo-Dioulasso. Or les éleveurs membres ne recevaient pas d'indemnités de transport. Par conséquent, il est probable que les éleveurs les plus éloignés aient assisté moins souvent à ces réunions et que les élus qui pouvaient venir facilement à Bobo-Dioulasso aient eu des échanges socio-techniques plus importants avec des partenaires variés. Ainsi quatre pédiluves ont été mis en place dans l'OPE de Yegueresso (figure 1) où résidait le président.

Les deux variables de perception figurant parmi les dix variables les plus importantes ont été, d'une part, une variable d'appréciation de l'efficacité de l'outil contre les tiques, généralement considérée par les chercheurs comme la première cause d'utilisation du traitement épicutané du bétail (15) et, d'autre part, l'appréciation de la facilité de mise en œuvre de la méthode. Malheureusement, ce ne sont pas des connaissances préalables et elles ne peuvent être utilisées pour choisir de futurs bénéficiaires en fonction de leurs conceptions.

Les modalités de la mise en place, décrites par la nature du parc d'attente, la nature de la mise en place du service, la distance pédiluve parc, les difficultés techniques et les difficultés financières apparaissent prépondérantes dans cette étude : la nature du parc d'attente a été la variable influençant le plus le nuage de points ! En effet, selon les critères de Mendras et Forsé (23), le critère impliqué est ici la complexité de la méthode. L'inventeur de la méthode avait déjà bien conscience de limiter les contraintes pratiques, par exemple en recommandant le respect d'une faible distance pédiluve parc (33). Les éleveurs traditionnels qui n'ont pas été encadrés par la recherche n'ont pas bénéficié d'un réel suivi. Au sein de l'APLL, non seulement les éleveurs étaient mieux équipés individuellement et étaient donc habitués à manipuler davantage de technologies différentes, mais ils ont en outre bénéficié d'un meilleur soutien technique puisqu'ils ont presque été tous suivis par un technicien. Il apparaît aussi que les suivis effectués par la recherche ont été particulièrement avantageux pour les éleveurs traditionnels, car outre l'appui technique, une aide financière dégressive a été apportée, ce qui a diminué largement la prise de risque des éleveurs. Un autre élément important qui n'apparaît pas dans l'étude était le fait que les deux pédiluves mis en place par la recherche étaient gérés de manière familiale : à Tondogosso, les éleveurs bénéficiaires et gestionnaires appartenaient à la même famille (dont deux frères) ; à Borodougou, les deux principaux utilisateurs étaient père et fils (le fils était le gérant). Les pédiluves du groupe 1 ont tous été gérés de manière individuelle, ce qui a diminué la nécessité d'adapter les pratiques de gestion.

Les parcs d'attente ont été de nature très différente entre les trois groupes et cela a eu un impact important. Ainsi la configuration la plus favorable a été l'utilisation de la stabulation comme parc d'attente, comme cela a été le cas pour le groupe 1. La totalité du groupe 2 a présenté des parcs d'attente ronds avec du grillage, ce qui a entraîné un fort pourcentage de difficultés techniques, en particulier des difficultés de passage non surmontées. Cette configuration apparaît donc particulièrement inadaptée, car elle se traduit par une mauvaise perception par les bovins du chemin à emprunter, entraînant un affolement dans le troupeau qui n'est plus canalisé vers la sortie par le pédiluve. Le caractère transparent du grillage est une source de stress pour des animaux habitués aux parcs de nuits faits de branchages (figure 6). Ainsi les éleveurs, en modulant différentes conformations d'efficacité variable, ont montré que le parc d'attente était une innovation en soi.

L'analyse des difficultés financières est délicate puisque cette variable peut être cause ou conséquence de non-adoption. Si la gestion financière d'un bien collectif est un problème en soi, cette variable devient agent causal et c'est bien une modalité de la mise en place sur laquelle un travail doit être réalisé. Si les éleveurs refusent de payer le service car ils n'ont pas adopté la méthode pour d'autres raisons, alors les difficultés financières sont un indicateur d'adoption. Il semble que les deux aspects de cette variable doivent être retenus et qu'il ne soit pas possible de les quantifier relativement dans cette étude.

Le caractère individuel ou collectif d'un pédiluve a déterminé de manière importante l'adoptabilité de la méthode car la gestion a été plus facile et les enjeux sociologiques ont été bien moindres dans le cas d'un usage individuel.
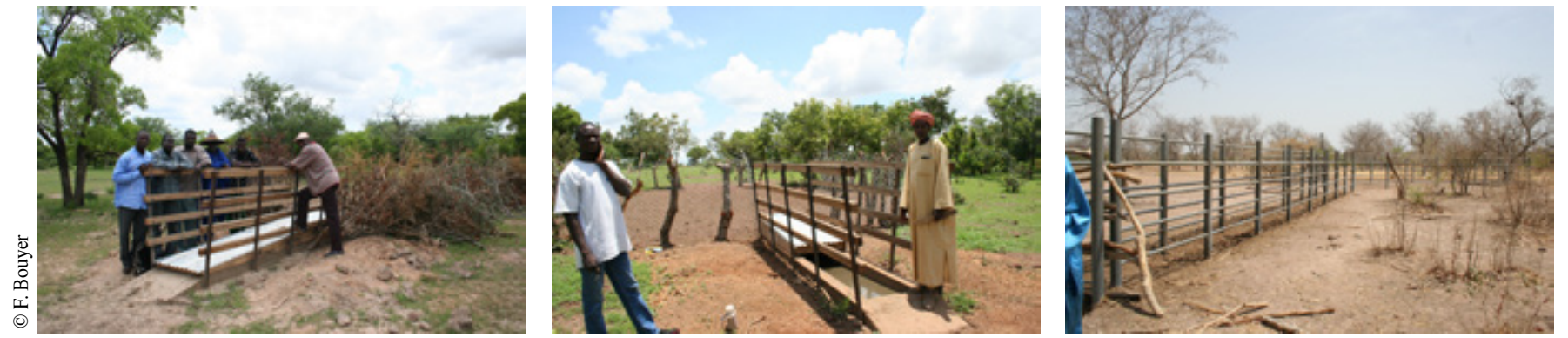

Figure 6 : à gauche, pédiluve avec parc d'attente traditionnel en entonnoir ; au centre, pédiluve avec parc d'attente rond avec grillage ; à droite, parc de vaccination métallique. 
Il était intéressant de noter l'importance du système d'élevage (décrit par la race des bovins, l'utilisation de parc métallique, la quantité de matériel individuel, le type d'activités menées par l'OPE) car ces éléments pouvaient être utilisés pour prédire l'adoption en fonction du type d'élevage. Le type d'activités menées par l'OPE indiquait le dynamisme du système de production, ses capacités de gestion et d'encadrement des éleveurs. Alors que l'APLL a trouvé des ressources internes pour suivre techniquement les pédiluves, cela n'a pas été possible pour les éleveurs de l'UEPL. Dans ce cas, en absence d'un suivi de qualité (comme celui apporté par la recherche), les difficultés à surmonter sont devenues limitantes. La logique économique ne permet pas d'expliquer l'abandon de la méthode puisque, dans toutes les localités où le nombre de passages est resté inférieur à 4000 , le produit insecticide n'a pas dû être renouvelé (car non épuisé) et puisque l'usage du pédiluve à Dafinso a été stimulé par la présence de la recherche pour un essai d'efficacité. Il est donc tentant de citer Alary (1) : «Mais les facteurs structurels et les logiques économiques ne peuvent expliquer la totalité du processus d'adoption. Le soutien social, voire moral, assuré par les agents de développement et les chercheurs a eu son rôle aussi ». Dans certaines OPE des blocages sociologiques non explicités interviennent et nous pouvons encore évoquer "la méfiance entre producteurs [qui] empêche les échanges intra-communautaires sans l'intervention d'agents extérieurs » (1).

En ce qui concerne la race des bovins, on pourrait penser que cette variable est juste associée à un type d'élevage qu'elle décrit et que ce sont d'autres caractéristiques des élevages modernes (comme la quantité de matériel individuel) qui ont une incidence directe sur l'adoption. Cependant, selon le témoignage d'un éleveur de l'UEPL, les métis n'ont pas présenté de difficultés de passage contrairement aux zébus Peuhls du même troupeau dont seulement la moitié de l'effectif a réussi à passer dans le pédiluve la première année d'usage. D'autres commentaires d'éleveurs suggèrent que les zébus Peuhls ont été moins dociles et donc qu'il a été plus difficile de les habituer à une telle infrastructure. Ceci n'est pas étonnant car les races européennes ont été sélectionnées sur la base de leur docilité (24), contrairement à la race zébu Peuhl (3). Il ne faut donc pas sous-estimer l'importance de ce paramètre puisque les difficultés de passage ont été évoquées fréquemment comme source de découragement des éleveurs traditionnels. Le paramètre « utilisation habituelle d'un parc métallique (stabulation ou parc de vaccination) » est remarquable puisqu'il pourrait avoir une valeur prédictive. Cette variable est associée à l'adoption de la méthode. On peut ici invoquer un apprentissage des animaux habitués à passer dans des dispositifs constitués de matériaux modernes (figure 6).

\section{Adoption et estimation du risque par les éleveurs}

L'avantage relatif du pédiluve par rapport aux autres méthodes de lutte (premier critère de Mendras et Forsé ; 23) a été évalué en station et sur le terrain : cette méthode a été moins coûteuse, efficace et pratique. Les variables « difficultés techniques », " difficultés de passage », " effet sur les tiques » et " facilité de mise en œuvre » ont participé à l'évaluation de ce critère et ont révélé que cet avantage différait d'un groupe d'éleveurs à l'autre car il dépendait de la mise en œuvre. L'essayabilité du pédiluve (quatrième critère) était faible, car seuls les éleveurs à proximité pouvaient l'essayer donc elle ne pouvait pas précéder la construction. Son observabilité chez autrui a été modérée mais doit être évoquée pour expliquer l'existence de quatre pédiluves dans une même OPE (Yegueresso). Enfin, la compatibilité par rapport au système en place et la complexité de la méthode (deuxième et troisième critères) ont été évaluées ensemble par toutes les autres variables qui décrivaient soit le système de production, soit les paramètres sociotechniques (comme le type d'activités menées par l'OPE). En fait, il n'était pas possible de donner une évaluation objective de chaque critère qui aurait été spécifique au pédiluve puisque ces variables variaient d'un groupe d'éleveurs à l'autre, pour lesquels les paramètres limitants n'étaient pas les mêmes. Par exemple, les difficultés de passage représentaient une contrainte importante pour les éleveurs des groupes traditionnels mais pas du tout pour les éleveurs modernes.

Les bons résultats d'adoption dans le groupe des éleveurs modernes de Ouagadougou n'ont pas été surprenants car les éleveurs étaient déjà entrés dans une démarche d'intensification de manière volontaire : ils avaient investi déjà dans du matériel moderne (stabulation métallique, couloir de vaccination, par exemple), quelquefois coûteux. L'installation du pédiluve n'a pas représenté pour cette catégorie d'éleveurs un risque important tant au niveau économique que technique ou social. L'usage individuel de l'infrastructure a induit une absence d'impact au niveau social. En revanche, pour les éleveurs traditionnels, le pédiluve a engendré un risque plus important. En effet, au niveau économique, les éleveurs ont évoqué le fait que les animaux ne pouvaient être traités par le pédiluve pendant la transhumance. Par conséquent, il est possible qu'ils aient sous-évalué le bénéfice économique et pratique du pédiluve. Les éleveurs ont investi financièrement dans les pédiluves de manière modérée. En revanche, la nécessité d'habituer les animaux au passage dans le pédiluve ainsi que la maîtrise technique (dosage du produit, remplissage des fiches de passage, etc.) demandaient un investissement en termes de temps et de travail non négligeable.

Enfin, l'usage collectif des pédiluves a eu un impact social. En effet, les gérants avaient un rôle stratégique puisqu'ils étaient responsables de l'entretien technique du pédiluve, et qu'ils devaient assister à tous les passages, connaître le nombre de bovins traités de chaque éleveur utilisateur et effectuer les calculs de paiement. Or ces gérants devaient être disponibles (c'était contraignant), de préférence lettrés, capables de comprendre les documents de gestion (abaques, fiches de passage). Par conséquent, des jeunes gens qui étaient allés à l'école étaient souvent choisis comme gérants plutôt que des notables d'un certain âge. Ils étaient choisis pour leur position d'obligation envers leurs aînés mais leur nouveau rôle leur a donné une nouvelle position stratégique susceptible de changer les relations sociales traditionnelles. Certains éleveurs ont ainsi déclaré que la gestion financière collective était difficile à envisager car «on ne paie pas ses enfants».

Plus le système de production est traditionnel et soumis à des conditions difficiles, plus il est basé sur des relations de solidarité fortes, bien établies, visant à garder la pérennité et la stabilité des entreprises familiales. Toute modification du système social est donc envisagée comme un stress important qui pèse lourd dans la balance du risque de l'innovation.

\section{- CONCLUSION}

Afin de favoriser l'adoption du pédiluve, celui-ci doit être recommandé aux éleveurs modernes ou en voie d'intensification, en particulier ceux utilisant des races métissées, un parc métallique et du matériel individuel varié. Une gestion individuelle est préférable mais peut être étendue aux membres d'une famille travaillant ensemble. Le parc d'attente doit être construit à la suite de la stabulation pour les éleveurs modernes, en forme d'entonnoir et avec des matériaux semblables à ceux du parc de nuit pour les autres. Enfin, en milieu traditionnel, un appui technique et organisationnel prolongé sur plusieurs années est fortement recommandé. 
La pertinence des critères de Mendras et Forsé dépend de la manière dont on les interprète car ils sont très généraux et un réel travail d'adaptation au contexte est nécessaire pour en extraire des critères précis et adaptés à l'étude. Ainsi « l'avantage relatif apporté par l'innovation par rapport à la situation initiale» et «la compatibilité par rapport au système en place » peuvent être analysés avec diverses variables techniques, économiques et sociologiques. Des connaissances globales, précises et multidisciplinaires de l'innovation à étudier sont nécessaires : c'est par exemple la connaissance de terrain des aspects zootechniques spécifiques qui a suggéré l'influence probable de la race des bovins, de la nature du parc d'attente ou de l'usage de parc métallique. La nature du suivi externe, les difficultés de paiement ou techniques, le niveau d'instruction, le niveau d'équipement sont des variables facilement transposables pour différentes innovations. Enfin, les deux variables de perception importantes concernent l'appréciation de deux aspects fondamentaux de l'innovation : son efficacité (ici uniquement vis-à-vis des tiques) et sa facilité de mise en œuvre. Rogers (28) souligne le fait qu'il importe de ne pas analyser uniquement les avantages objectifs mais bien les perceptions de ces avantages. Cet élément semble primordial, en particulier dans les études portant sur le domaine agricole en Afrique de l'Ouest.

Les résultats obtenus dans cette étude seront importants à intégrer dans les futures campagnes de contrôle des glossines et des trypanosomoses désireuses d'impliquer les populations bénéficiaires dans la lutte de façon durable (6).

\section{Remerciements}

Ce travail a bénéficié de l'appui du Coraf et du Cirad pour le financement de l'étude. Nous sommes très reconnaissants envers le directeur général du Cirdes, Pr Abdoulaye Gouro, pour les excellentes conditions de travail apportées. Nous remercions particulièrement M. Lassina Sanogo pour la qualité du travail de terrain effectué.

\section{BIBLIOGRAPHIE}

1. ALARY V., 2006. L'adoption de l'innovation dans les zones agropastorales vulnérables du Maghreb. Afr. contemp., 219 : 81-101.

2. AUBREVILLE A., 1950. Flore forestière soudano-guinéenne. Paris, France, Société d'éditions géographiques maritimes et coloniales, 525 p.

3. AYANTUNDE A., KANGO M., HIERNAUX P., UDO H., TABO R., 2007. Herders' perceptions on ruminant livestock breeds and breeding management in Southwestern Niger. Hum. Ecol., 35: 139-149.

4. BAUER B., KABORE I., LIEBISCH A., MEYER F., PETRICH-BAUER J., 1992. Simultaneous control of ticks and tsetse flies in Satiri, Burkina Faso, by the use of flumethrin pour on for cattle. Trop. Med. Parasitol., 43: 41-46.

5. BOUYER J., BENGALY Z., 2006. Evaluation de la situation entomologique et épidémiologique en vue de l'élaboration d'un plan de lutte contre les trypanosomoses animales et leur vecteur dans la zone d'intervention du PAEOB. Bobo-Dioulasso, Burkina Faso, Cirdes/Cirad, $30 \mathrm{p}$.

6. BOUYER F., HAMADOU S., ADAKAL H., LANCELOT R., STACHURSKI F., BELEM A.M.G., BOUYER J., 2011. Restricted application of insecticides: a promising tsetse control technique, but what do the farmers think of it? PLoS Negl trop Dis., 5: e1276.

7. BOUYER I., KABORE I., STACHURSKI F., DESQUESNES M., 2005. Epicutaneous treatment of cattle. Santé animale en Afrique de I'Ouest, Recommandations techniques. Bobo-Dioulasso, Burkina Faso, Cirdes/ Cirad, $8 \mathrm{p}$

8. BOUYER J., STACHURSKI F., GOURO A., LANCELOT R., 2009. Control of bovine trypanosomosis by restricted application of insecticides to cattle using footbaths. Vet Parasitol., 161: 187-193.

9. BOUYER J., STACHURSKI F., GOURO A.S., LANCELOT R., 2008. Traitement insecticide des bovins contre les glossines par pédiluve en conditions expérimentales. Revue Elev. Méd. vét. Pays trop., 61 : 161167.

10. BOUYER J., STACHURSKI F., KABORE I., BAUER B., LANCELOT R., 2007. Tsetse control in cattle from pyrethroid footbaths. Prev. vet. Med., 78: $223-238$

11. CHIA E., 2004. Principes, méthodes de la recherche en partenariat: une proposition pour la traction animale. Revue Elev. Méd. vét. Pays trop., $\mathbf{5 7}:$ :233-240.

12. CONOVER W.J., 1971. one-sample "Kolmogorov" test, two-sample "Smirnov" test. Practical nonparametric statistics. New York, USA, John Wiley, p. 295-314.
13. COURTIN F., RAYAISSE J.-B., TAMBOURA I., SERDEBEOGO O., KOUDOUGOU Z., SOLANO P., SIDIBE I., 2010. Updating the Northern tsetse limit in Burkina Faso (1949-2009): Impact of global change. Int. J. Environ. Res. Publ. Health, 7: 1708-1719.

14. HAMADOU S., MARICHATOU H., KAMUANGA M., KANWE B.A., SIDIBE A.G., PARE J., DIOUARA H., SANGARE M.I., SANOGO O., 2004. Diagnostic des systèmes de production laitière en Afrique de I'Ouest : typologie des élevages périurbains. In : Conf. int./région. Elevage en Afrique de I'Ouest et du Centre, Banjul, Gambie, 8-12 nov. 2004

15. HARGROVE J.W., 2003. Tsetse eradication: sufficiency, necessity and desirability. Edinburgh, GB, DFID, Animal Health Programme, 134 p.

16. HERITIER F., 2001. Innovation, invention, découverte. In : Actes $12^{\mathrm{e}}$ Festival int. Géographie, Saint-Dié-des-Vosges, France, 4-7 oct. 2001, p. 11.

17. HOLLANDER M., WOLFE D.A., 1973. Non parametric statistical inference. New York, USA, John Wiley, $344 p$.

18. ITARD J., CUISANCE D., TACHER G., 2003. Trypanosomoses : historique - répartition géographique. In : Lefèvre P.-C., Blancou J., Chermette R., éds, Principales maladies infectieuses et parasitaires du bétail. Europe et régions chaudes. Paris, France, Lavoisier, p. 1607-1615.

19. JOUVE P., 1989. Cours international. Systèmes agraires et stratégies de développement. Montpellier, France, Cirad-DSA, $100 \mathrm{p}$.

20. KAMUANGA M., 2003. Socio-economic and cultural factors in the research and control of trypanosomiasis. PAAT Technical and Scientific Series. Rome, Italy, FAO, 73 p.

21. KAMUANGA M., SIGUE H., SWALLOW B., BAUER B., D'IETEREN G., 2001. Farmers' perceptions of the impact of tsetse and trypanosomosis control on livestock production: Evidence from Southern Burkina Faso. Trop. Anim. Health Prod., 33: 141-153.

22. LEFORT J., 1988. Innovation technique et expérimentation en milieu paysan. Cah. Rech. Dév., 17 : 1-10.

23. MENDRAS H., FORSE M., 1983. Le changement social. Paris, France, Armand Colin, 284 p.

24. MIGNON-GRASTEAU S., BOISSY A., BOUIX J., FAURE J.-M. FISHER A.D., HINCH G.N., JENSEN P., LE NEINDRE P., MORMEDE P., PRUNET P., VANDEPUTTE M., BEAUMONT C., 2005. Genetics of adaptation and domestication in livestock. Livest. Prod. Sci., 93: 3-14.

25. MINISTERE DES RESSOURCES ANIMALES, MINISTERE DE L'ECONOMIE ET DU DEVELOPPEMENT, 2004. Enquête nationale sur les effectifs du cheptel. Ouagadougou, Burkina Faso, 85 p.

26. MUNZEL U., HOTHORN L.A., 2001. A unified approach to simultaneous rank test procedures in the unbalanced one-way layout. Biom. J., 43: 553-569. 
27. R DEVELOPMENT CORE TEAM, 2010. R: A language and environment for statistical computing. Vienna, Austria, R Foundation for Statistical Computing. http://www.R-project.org

28. ROGERS E., 1983. Diffusion of innovations. New York, USA, Free Press, $576 \mathrm{p}$.

29. SCHUMPETER B., 1935. Théorie de l'évolution économique. Recherche sur le profit, l'intérêt et le cycle de la conjoncture. Paris, France, Dalloz.

30. STACHURSKI F., 2000. Invasion of West African cattle by the tick Amblyomma variegatum. Med. Vet. Entomol., 14: 391-399.

31. STACHURSKI F., 2000. Modalités de la rencontre entre la stase adulte de la tique Amblyomma variegatum (Acari, Ixodida) et les bovins: applications potentielles à la lutte contre ce parasite. Thèse Doct, Université Montpellier II, Montpellier, France, 264 p.

\section{Summary}

Bouyer F., Belem A., Seyni H., Adakal H., Lancelot R., Stachurski F., Bouyer J. Adoption of a new disease control method by farmers: the acaricide/insecticide footbath in Burkina Faso

The acaricide/insecticide footbath is a new integrative vector control method in subhumid areas of West Africa: its efficiency was validated through many experimental and field studies. This exogenous health-related invention was proposed by researchers, co-built with herder groups ten years ago, then disseminated. In total, 22 footbaths and 72 herders were surveyed. Based on 97 variables concerning sociology, organization, livestock system, tool appraisal and implementation modalities, 21 were considered as active and seven as indicators of adoption after preliminary analyses. The multivariate analyses carried out on these variables characterized three herder groups whose adoption was assessed. The first group consisted of the modern herders of Ouagadougou who had adopted the method well. The more traditional herders of Bobo-Dioulasso were split into two groups: the first one adopted the footbath, the second did not. The ten main variables that discriminated the groups were analyzed. They related to the livestock system, implementation modalities and tool appraisal. Risk assessment of innovation by herders was discussed with the evaluation criteria of adoptability from Mendras and Forsé. This analysis highlights the variations between individual perceptions of benefits and risks, and the predominant role of the social technical network.

Keywords: Innovation - Rearing system - Insect control - Mite control - Intensification - West Africa.
32. STACHURSKI F., BOUYER J., BOUYER F., 2005. Lutte contre les ectoparasites des bovins par pédiluve : méthode innovante utilisée en zone périurbaine subhumide du Burkina Faso. Revue Elev. Méd. vét. Pays trop., $58: 221-228$.

33. STACHURSKI F., LANCELOT R., 2006. Foot-bath acaricide treatment to control cattle infestation by the tick Amblyomma variegatum. Med. Vet. Entomol., 20: 402-412.

34. TENENHAUS M., YOUNG F.W., 1985. An analysis and synthesis of multiple correspondence analysis, optimal scaling, dual scaling, homogeneity analysis and other methods for quantifying categorical multivariate data. Psychometrika, 50: 91-119.

Mis en ligne en juin 2013

\section{Resumen}

Bouyer F., Belem A., Seyni H., Adakal H., Lancelot R., Stachurski F., Bouyer J. Adopción de un nuevo método de lucha sanitaria en medio campesino: el pediluvio acaricida/insecticida en Burkina Faso

El pediluvio acaricida/insecticida es un nuevo método de lucha integrada contra los vectores en zona la sub húmeda del Oeste africano: su eficacidad fue validada mediante numerosos estudios en situaciones experimentales y reales. Esta invención sanitaria de origen exógeno (propuesta por la investigación) fue co-construida con grupos de criadores hace diez años, siendo consecuentemente difundida. En total, se estudiaron 22 pediluvios y 72 criadores. A partir de 97 variables de adopción, concerniendo sociología, aspectos de organización, sistema de cría, apreciación del método y las modalidades de empleo, a partir de análisis preliminares, 21 fueron consideradas como activas y siete como indicadores de adopción. Fueron luego sometidas a análisis multi-variados, permitiendo caracterizar tres gropos de criadores, cuya adopción fue evaluada. El primer grupo estuvo constituido por los criadores modernos de Ouagadougou, quienes adoptaron bien el método. Los criadores más tradicionales de Bobo-Dioulasso fueron separados en dos grupos, uno no adoptó el sistema de pediluvio, contrariamente al segundo. Se analizaron las diez variables principalmente discriminantes de estos grupos. Estas siguieron el sistema de cría, las modalidades de implementación del método y la apreciación del método. Se discute la apreciación del riesgo de la innovación percibida por los criadores, gracias a los criterios de evaluación de la adaptabilidad de Mendras y Forsé Este análisis realzó las variaciones de percepción individuales de los beneficios y de los riesgos, así como el papel predominante del la red socio-técnica

Palabras clave: Innovación - Systema de cría - Control de insectos - Control de ácarios - Intensificación - Africa Occidental. 